\title{
Bounds for Domination Parameters in Cayley Graphs on Dihedral Group
}

\author{
T. Tamizh Chelvam, G. Kalaimurugan \\ Department of Mathematics, Manonmaniam Sundaranar University, Tirunelveli, India \\ Email: tamche59@gmail.com
}

Received November 3, 2011; revised December 18, 2011; accepted December 25, 2011

\begin{abstract}
In this paper, sharp upper bounds for the domination number, total domination number and connected domination number for the Cayley graph $G=\operatorname{Cay}\left(D_{2 n}, \Omega\right)$ constructed on the finite dihedral group $D_{2 n}$, and a specified generating set $\Omega$ of $D_{2 n}$. Further efficient dominating sets in $G=\operatorname{Cay}\left(D_{2 n}, \Omega\right)$ are also obtained. More specifically, it is proved that some of the proper subgroups of $D_{2 n}$ are efficient domination sets. Using this, an E-chain of Cayley graphs on the dihedral group is also constructed.
\end{abstract}

Keywords: Cayley Graph; Dihedral Group; Domination; Total Domination; Connected Domination; Efficient Domination

\section{Introduction and Notation}

Design of interconnection networks is an important integral part of any parallel processing of distributed system. There has been a strong interest recently in using Cayley graphs as a model for developing interconnection networks for large interacting arrays of CPU's. An excellent survey of interconnection networks based on Cayley graphs can be found in [1]. The concept of domination for Cayley graphs has been studied by various authors [2-7]. I. J. Dejter and O. Serra [3] obtained efficient dominating sets for Cayley graphs constructed on a class of groups containing permutation groups. The efficient domination number for vertex transitive graphs has been obtained by Jia Huang and Jun-Ming Xu [4]. A necessary and sufficient condition for the existence of an independent perfect domination set in Cayley graphs has been obtained by J. Lee [5]. Total domination in graphs was introduced by Cockayne, Dawes, and Hedetniemi [2] and is now well studied in graph theory. T. Tamizh Chelvam and I. Rani [6-8] have obtained bounds for various domination parameters for a class of Circulant graphs.

Let $\Gamma$ be a finite group. Let $\Omega$ be a generating set of $\Gamma$ satisfying $e \notin \Omega$ and $a \in \Omega$ implies $a^{-1} \in \Omega$. The Cayley graph corresponding to $\Gamma$ is the graph $G=(V, E)$, where $V(G)=\Gamma$ and $E(G)=\{(x, x a): x \in V(G), a \in \Omega\}$ and it is denoted by $G=\operatorname{Cay}(\Gamma, \Omega)$. Let $G=(V, E)$, be a finite, simple and undirected graph. We follow the terminology of [9]. A set $S \subseteq V$ of vertices in a graph $G$ is called a dominating set if every vertex $v \in V$ is either an element of $S$ or adjacent to an element of $S$. A dominating set $S$ is a minimal dominating set if no proper subset of $S$ is a dominating set. The domination number $\chi(G)$ of a graph $G$ is the minimum cardinality of a dominating set in $G$ and the corresponding dominating set is called a $\gamma$-set. A set $S \subseteq V$ is called a total dominating set if every vertex $v$ $\in V$ is adjacent to an element $u(\neq v)$ of $S$. The total domination number $\gamma_{t}(G)$ equals the minimum cardinality among all the total dominating sets in $G$ and the corresponding total dominating set is called a $\gamma_{t}$-set. A dominating set $S$ is called a connected dominating set if the induced subgraph $\langle S\rangle$ is connected. The connected domination number $\gamma_{c}(G)$ of a graph $G$ equals the minimum cardinality of a connected dominating set in $G$ and a corresponding connected dominating set is called a $\gamma_{\mathrm{c}}$-set. A set $S \subseteq V$ is called an efficient dominating set (E-set) if for every vertex $v \in V,|N[v] \cap S|=1$.

An E-chain is a countable family of nested graphs, each of which has an E-set. We say that a countable family of graphs $\boldsymbol{G}=\left\{G_{i}, i \geq 1\right\}$ with each $G_{i}$ has an E-set $S_{i}$ is an inclusive E-chain if for every $i \geq 1$, there exists a surjective map $f_{i}: G_{i+1} \rightarrow G_{i}$ such that $f_{i}^{-1}\left(S_{i}\right) \subset S_{i+1}$. And also we define that a finite family of graphs $\boldsymbol{G}=\left\{G_{i}\right.$, $i \geq 0\}$ is an inductive E-chain if every $G_{i+1}$ is a spanning subgraph of $G_{i}$ and each $G_{i}$ has an E-set $S_{i}$. Let $V\left(G_{i}\right)$ be any finite group and if, for each $i \geq 0$, there exists a bijective map $\zeta_{i}: V\left(G_{i}\right) \rightarrow V\left(G_{i+1}\right)$ such that $\zeta_{i}\left(S_{i}\right) \subseteq \mathrm{S}_{\mathrm{i}+1}$ and $S_{i}$ is the subgroup of $V\left(G_{i}\right)$ then we say that $\boldsymbol{G}$ is an inductive subgroups E-chain.

A graph $\tilde{G}$ is called a covering of $G$ with projection $p: \tilde{G} \rightarrow G$ if there is a surjection $p: V(\tilde{G}) \rightarrow V(G)$ 
such that $p /{ }_{N(\tilde{v})}: N(\tilde{v}) \rightarrow N(v)$ is a bijection for any vertex $v \in V(G)$ and $\tilde{v} \in p^{-1}(v)$. We use the covering function to show the inclusive E-chain.

In this paper, we obtain upper bounds for domination number, total domination number and connected domination number in a Cayley graph $G=\operatorname{Cay}\left(D_{2 n}, \Omega\right)$ constructed on the dihedral group $D_{2 n}$, for $n \geq 3$ and a generating set $\Omega$. Further, we obtain some E-sets in $G=\operatorname{Cay}\left(D_{2 n}, \Omega\right)$. Note that the dihedral group $D_{2 n}$ with identity $e$ is the group generated by two elements $r$ and $s$ with $o(r)=n, o(s)=2$ and $r s=s r^{-1}$. From these defining relations, one can take

$D_{2 n}=\left\{e, r, r^{2}, r^{3}, \cdots, r^{n-1}, s, s r, s r^{2}, \cdots, s r^{n-1}\right\}$ and $G=\operatorname{Cay}\left(D_{2 n}, \Omega\right)$, where $\Omega$ is a generating set of $D_{2 n}$. Throughout this paper, $n \geq 3$ be an integer, $\Gamma=D_{2 n}$, $m=\frac{n-1}{2}$ and $k, t$ be integers such that $1 \leq k \leq m$,

$1 \leq t \leq n$. We take the generating set $\Omega$ in the form that $\Omega=$

$\left\{r^{a_{1}}, r^{a_{2}}, \cdots, r^{a_{k}}, r^{n-a_{k}}, r^{n-a_{k-1}}, \cdots, r^{n-a_{1}}, s r^{b_{1}}, s r^{b_{2}}, \cdots, s r^{b_{t}}\right\}$,

where $1 \leq a_{1}<a_{2}<\cdots<a_{k} \leq m$ and

$0 \leq b_{1}<b_{2}<\cdots<b_{t} \leq n-1$. Let $d_{1}=a_{1}, d_{i}=a_{i}-a_{i-1}$ for $2 \leq i \leq k, \quad d_{1}^{\prime}=b_{1}, d_{j}^{\prime}=b_{j}-b_{j-1} \quad$ for $\quad 2 \leq j \leq t \quad$ and $d=\max _{1 \leq i \leq k, 1 \leq j \leq t}\left\{d_{i}, d_{j}^{\prime}\right\}$. Some of the results are listed below for further reference.

Theorem 1 [4] Let $G$ be a k-regular graph. Then

$\gamma(G) \geq \frac{|V(G)|}{k+1}$, with the equality if and only if $G$ has an efficient dominating set.

Theorem 2 [5] Let $p: \tilde{G} \rightarrow G$ be a covering and let $S$ be a perfect domination set of $G$. Then $p^{-1}(S)$ is a perfect domination set of $\tilde{G}$. Moreover, if $S$ is independent, then $p^{-1}(S)$ is independent.

Theorem 3 [10] Every subgroup of the dihedral group $D_{2 n}$ is cyclic or dihedral. A complete listing of the subgroups is as follows:

1) cyclic subgroups $\left\langle r^{d}\right\rangle$, where $d$ divides $n$, with in$\operatorname{dex} 2 d$.

2) dihedral subgroups $\left\langle r^{d}, r^{i} s\right\rangle$, where $d$ divides $n$ and $0 \leq i \leq d-1$ with index $d$. Every subgroup of $D_{2 n}$ occurs exactly once in this listing.

\section{Domination, Total Domination and Connected Domination Numbers}

In this section, we obtain upper bounds for the domination number, total domination number and connected domination number of graph $G=\operatorname{Cay}\left(D_{2 n}, \Omega\right)$. Also whenever the equality occurs we give the corresponding sets.

Lemma 4 Let $n \geq 3$ be an integer, $m=\frac{n-1}{2}$ and $k$, t are integers such that $1 \leq k \leq m, 1 \leq t \leq n$. Let

$\Omega=$

$\left\{r^{a_{1}}, r^{a_{2}}, \cdots, r^{a_{k}}, r^{n-a_{k}}, r^{n-a_{k-1}}, \cdots, r^{n-a_{1}}, s r^{b_{1}}, s r^{b_{2}}, \cdots, s r^{b_{t}}\right\}$

and $G=\operatorname{Cay}\left(D_{2 n}, \Omega\right)$. If $d_{1}=a_{1}, d_{i}=a_{i}-a_{i-1}$ for $2 \leq i \leq k, \quad d_{1}^{\prime}=b_{1}, d_{j}^{\prime}=b_{j}-b_{j-1}$ for $2 \leq j \leq t$ and $d=\max _{1 \leq i \leq k, 1 \leq j \leq t}\left\{d_{i}, d_{j}^{\prime}\right\}$, then

$\gamma(G) \leq 2 d \frac{n}{2 d+2 a_{k}+b_{t}-b_{1}}$.

Proof. Let $x=2 a_{k}+2 d+b_{t}-b_{1}$ and $l=\left\lceil\frac{n}{x}\right\rceil$. Consider the set

$$
\begin{aligned}
& S= \\
& \left\{r^{i x+g}, s r^{n-\left(a_{k}+d-b_{1}+i x+g\right)}: 0 \leq i \leq l-1 \text { and } 0 \leq g \leq d-1\right\} .
\end{aligned}
$$

Clearly $|S|=2 d l$ and

$$
N[S]=\bigcup_{i=0}^{l-1}\left\{N\left[r^{i x+g}\right] \cup N\left[s r^{n-\left(a_{k}+d-b_{1}+i x+g\right)}\right]\right\},
$$

where $0 \leq i \leq l-1$ and $0 \leq g \leq d-1$ We have to prove that $V(G) \subseteq N[S]$. If $v \in V(G)$, then we can write $v$ as either one vertex of the form $v=r^{c}$ or $v=s r^{n-\left(c-b_{t}\right)}$, where $0 \leq c \leq n-1$. By the division algorithm, $c=x i+j$, where $0 \leq i \leq l-1$ and $0 \leq j \leq x-1$.

Suppose $v=r^{c}$. We have the following cases:

Case 1. Suppose $0 \leq i \leq l-1$ and $0 \leq j \leq a_{k}+d-1$.

Subcase 1.1 If $0 \leq j<a_{1}$, then by the definition of $d, v \in S \subseteq N[S]$.

Subcase 1.2 If $j=a_{m}+g$, for some integers $m, g$ with $1 \leq m \leq k$ and $0 \leq g \leq d-1$ then $v=r^{i x+a_{m}+g}$ whereas $r^{i x+g} \in S$ and so $v \in N\left[r^{i x+g}\right] \subseteq N[S]$.

Case 2. Suppose $0 \leq i \leq l-1$ and $a_{k}+d \leq j \leq a_{k}+d+b_{t}-b_{1}+d-1$. In this case, there exists an integer $h$ with $1 \leq h \leq b_{t}-b_{1}+d-1$ such that $v \cdot s r^{h}=s r^{n-\left(a_{k}+d-b_{1}+i x\right)}$.

Subcase 2.1 If $h \in \Omega_{2}=\left\{b_{1}, b_{2}, \cdots, b_{t}\right\}$, then $v \in N\left(s r^{n-\left(a_{k}+d-b_{1}+i x\right)}\right) \subseteq N[S]$

Subcase 2.2 Suppose $h=b_{m}+g$, for some integers $m$, $g$ with $1 \leq m \leq t$ and $1 \leq g \leq d-1$. In this case, $v \cdot s r^{b_{m}}=s r^{n-\left(a_{k}+d-b_{1}+i x+g\right)}$, which means that $v \in S r^{n-\left(a_{k}+d-b_{1}+i x+g\right)} \subseteq N[S]$.

Case 3. Suppose $0 \leq i \leq l-2$ and

$$
a_{k}+d+b_{t}-b_{1}+d \leq j \leq a_{k}+d+b_{t}-b_{1}+d+a_{k}-1 \text {. }
$$

In this case, there exists an integer $h$ with $1 \leq h \leq a_{k}$ such that $v \cdot r^{h}=r^{(i+1) x}$.

Subcase 3.1 If $h \in \Omega_{1}=\left\{a_{1}, a_{2}, \cdots, a_{k}\right\}$, then $v \in N\left(r^{(i+1) x}\right) \subseteq N[S]$.

Subcase 3.2 Suppose $h=a_{m}-g$, for some integers $m$, $g$, with $1 \leq m \leq k$ and $1 \leq g \leq d-1$. In this case, $v \cdot r^{a_{m}}=r^{(i+1) x+g}$, which means that $v \in N\left(r^{(i+1) x+g}\right) \subseteq N[S]$. 
Case 4. Suppose $i=l-1$ and

$$
a_{k}+d+b_{t}-b_{1}+d \leq j \leq a_{k}+d+b_{t}-b_{1}+d+a_{k}-1 \text {. }
$$

Then there exists an integer $h$ with $1 \leq h \leq a_{k}$ such that $v \cdot r^{h}=r^{0}$.

Subcase 4.1 If $h \in \Omega_{1}$, then $v \in N\left(r^{0}\right) \subseteq N[S]$.

Subcase 4.2 Suppose $h=a_{m}-g$, for some integers $m$, $g$ with $1 \leq m \leq k$ and $1 \leq g \leq d-1$. In this case, $v \cdot r^{a_{m}}=r^{g}$ which means that $v \in N\left(r^{g}\right) \subseteq N[S]$.

Suppose $v=s r^{n-\left(c-b_{t}\right)}$. We have the following cases:

Case 1. Suppose $0 \leq i \leq l-1$ and $0 \leq j \leq b_{t}-b_{1}+d-1$. In this case, there exists an integer $h$ with $0 \leq h \leq b_{t}-b_{1}+d-1$ such that $v \cdot s r^{h}=r^{i x}$

Subcase 1.1 If $h \in \Omega_{2}$, then $v \in N\left(r^{i x}\right) \subseteq N[S]$.

Subcase 1.2 Suppose $h=b_{m}+g$, for some integers $m$, $g$ with $1 \leq m \leq t$ and $1 \leq g \leq d-1$. In this case, $v \cdot s r^{b_{m}}=r^{i x+g}$, which means that $v \in N\left(r^{i x+g}\right) \subseteq N[S]$.

Case 2. Suppose $0 \leq i \leq l-1$ and $b_{t}-b_{1}+d \leq j \leq b_{t}-b_{1}+d+a_{k}-1$. In this case, there exists an integer $h$ with $1 \leq h \leq a_{k}$ such that $v \cdot r^{h}=s r^{n-\left(a_{k}+d-b_{1}+i x\right)}$.

Subcase 2.1 If $h \in \Omega_{1}$ then $v \in N\left(s r^{n-\left(a_{k}+d-b_{1}+i x\right)}\right) \subseteq N[S]$.

Subcase 2.2 Suppose $h=a_{m}-g$, for some integers $m$, $g$ with $1 \leq m \leq k$ and $1 \leq g \leq d-1$. In this case,

$v \cdot r^{a_{m}}=s r^{n-\left(a_{k}+d-b_{1}+i x+g\right)}$, which means that $v \in s r^{n-\left(a_{k}+d-b_{1}+i x+g\right)} \subseteq N[S]$.

Case 3. Suppose $0 \leq i \leq l-1$ and $b_{t}-b_{1}+d+a_{k} \leq j \leq b_{t}-b_{1}+2 d+2 a_{k}-1$. In this case, there exists an integer $h$ with $0 \leq h \leq a_{k}+d-1$ such that $v \cdot r^{h}=s r^{n-\left(a_{k}+d-b_{1}+x i\right)}$.

Subcase 3.1 If $0 \leq h<a_{1}$, then by the definition of $d, v \in S \subseteq N[S]$.

Subcase 3.2 Suppose $h=a_{m}+g$, for some integers $m$, $g$ with $1 \leq m \leq k$ and $0 \leq g \leq d-1$. In this case, $v \cdot r^{a_{m}}=s r^{n-\left(a_{k}+d-b_{1}+i x+g\right)}$, which means that $v \in s r^{n-\left(a_{k}+d-b_{1}+i x+g\right)} \subseteq N[S]$.

Thus $S$ is a dominating set of $G$.

The following lemma provides an upper bound for the total domination number in $G=\operatorname{Cay}\left(D_{2 n}, \Omega\right)$.

Lemma 5 Let $n \geq 3$ be an integer, $m=\left\lfloor\frac{n-1}{2}\right\rfloor$ and $k$, $t$ be integers such that $1 \leq k \leq m, 1 \leq t \leq n$. Let

$\Omega=$

$\left\{r^{a_{1}}, r^{a_{2}}, \cdots, r^{a_{k}}, r^{n-a_{k}}, r^{n-a_{k-1}}, \cdots, r^{n-a_{1}}, s r^{b_{1}}, s r^{b_{2}}, \cdots, s r^{b_{t}}\right\}$, and $G=\operatorname{Cay}\left(D_{2 n}, \Omega\right)$. If $d_{1}=a_{1}, d_{i}=a_{i}-a_{i-1}$ for $2 \leq i \leq k, d_{1}^{\prime}=b_{11}, d_{j}^{\prime}=b_{j}-b_{j-1}$ for $2 \leq j \leq t$ and $d=\max _{1 \leq i \leq k, 1 \leq j \leq t}\left\{d_{i}, d_{j}^{\prime}\right\}$, then $\gamma_{t}(G) \leq 2 d \frac{n}{d+2 a_{k}}$.

Proof. Let $x=d+2 a_{k}$ and $l=\left\lceil\frac{n}{x}\right\rceil$. Consider the set

$$
S_{t}=\left\{r^{i x+g}, s r^{n-\left(i x+g-b_{1}\right)}: 0 \leq i \leq l-\text { and } 0 \leq g \leq d-1\right\} .
$$

Clearly $\left|S_{t}\right|=2 d l$. We have to prove that $V(G) \subseteq N\left(S_{t}\right)$ If $v \in V(G)$, then we can write $v$ as either one vertex of the form $v=r^{c}$ or $v=s r^{n-\left(c-b_{1}\right)}$, where $0 \leq c \leq n-1$. By the division algorithm, $c=x i+j$, where $0 \leq i \leq l-1$ and $0 \leq j \leq x-1$. We have the following cases:

Case 1. Suppose $0 \leq i \leq l-1$ and $0 \leq j<a_{1}$. For some integer $g$ with $0 \leq g \leq d-1$ and by the definition of $d$, if $v=r^{c}$, then $v \in N\left(s r^{n-\left(i x+g-b_{1}\right)}\right) \subseteq N\left(S_{t}\right)$ or if $v=s r^{n-\left(c-b_{1}\right)}$, then $v \in N\left(r^{i x+g}\right) \subseteq N\left(S_{t}\right)$.

Case 2. Suppose $0 \leq i \leq l-1$ and $a_{1} \leq j<a_{k}+d-1$. We can write $j=a_{m}+g$, for some integers $m, g$ with $1 \leq m \leq k$ and $0 \leq g \leq d-1$. If $v=r^{c}$, then $v=r^{i x+g+a_{m}}$ whereas $r^{i x+g} \in S_{t}$ and so $v \in N\left(r^{i x+g}\right) \subseteq N\left(S_{t}\right)$ or if $v=s r^{n-\left(c-b_{1}\right)}$, then $v=s r^{n-\left(i x+g+a_{m}-b_{1}\right)}$ whereas $s r^{n-\left(i x+g-b_{1}\right)} \in S_{t}$ and so $v \in N\left(s r^{n-\left(i x+g-b_{1}\right)}\right) \subseteq N\left(S_{t}\right)$.

Case 3. Suppose $0 \leq i \leq l-2$ and $d+a_{k} \leq j<d+2 a_{k}$. In this case, there exists an integer $h$ with $1 \leq h \leq a_{k}$ such that $v \cdot r^{h}=r^{(i+1) x}$ or $v \cdot r^{h}=s r^{n-\left((i+1) x-b_{1}\right)}$.

Subcase 3.1 Suppose $h \in \Omega_{1}=\left\{a_{1}, a_{2}, \cdots, a_{k}\right\}$ and if $v=r^{c}$, then $v \in\left(r^{(i+1) x}\right) \subseteq N\left(S_{t}\right)$ or if $v=s r^{n-\left(c-b_{1}\right)}$, then $v \in N\left(s r^{n-\left((i+1) x-b_{1}\right)}\right) \subseteq N\left(S_{t}\right)$.

Subcase 3.2 Suppose $h=a_{m}-g$, for some integers $m$, $g$ with $1 \leq m \leq k$ and $1 \leq g \leq d-1$. In this case, if $v=r^{c}$, then $v \cdot r^{a_{m}}=r^{(i+1) x+g}$, which means that $v \in N\left(r^{(i+1) x+g}\right) \subseteq N\left(S_{t}\right)$ or if $v=s r^{n-\left(c-b_{1}\right)}$, then $v \cdot r^{a_{m}}=s r^{n-\left((i+1) x+g-b_{1}\right)}$, which implies that $v \in N\left(s r^{n-\left((i+1) x+g-b_{1}\right)}\right) \subseteq N\left(S_{t}\right)$.

Case 4. Suppose $i=l-1$ and $d+a_{k} \leq j<d+2 a_{k}$. Then there exists an integer $h$ with $1 \leq h \leq a_{k}$ such that $v \cdot r^{h}=r^{0}$ or $v \cdot r^{h}=s r^{n-b_{1}}$.

Subcase 4.1 When $h \in \Omega_{1}$, and if $v=r^{c}$, then $v \in N\left(r^{0}\right) \subseteq N\left(S_{t}\right)$ or if $v=s r^{n-\left(c-b_{1}\right)}$, then $v \in N\left(s r^{n-b_{1}}\right) \subseteq N\left(S_{t}\right)$.

Subcase 4.2 Suppose $h=a_{m}-g$, for some integers $m$, $g$ with $1 \leq m \leq k$ and $1 \leq g \leq d-1$. In this case, if $v=r^{c}$ and $v \cdot r^{a_{m}}=r^{g}$, which means that $v \in N\left(r^{g}\right) \subseteq N\left(S_{t}\right)$ or if $v=s r^{n-\left(c-b_{1}\right)}$, then $v \cdot r^{a_{m}}=s r^{n-\left(g-b_{1}\right)}$, which means that $v \in N\left(s r^{n-\left(g-b_{1}\right)}\right) \subseteq N\left(S_{t}\right)$.

Thus $S_{t}$ is a total dominating set of $G$. $\gamma_{t} \leq\left|S_{t}\right|=2 d l$.

Now we obtain an upper bound for the connected domination number. 
Lemma 6 Let $n \geq 3$ be an integer, $m=\left\lfloor\frac{n-1}{2}\right\rfloor$ and $k$, $t$ be integers such that $1 \leq k \leq m, 1 \leq t \leq n$. Let

$\Omega=$

$\left\{r^{a_{1}}, r^{a_{2}}, \cdots, r^{a_{k}}, r^{n-a_{k}}, r^{n-a_{k-1}}, \cdots, r^{n-a_{1}}, s r^{b_{1}}, s r^{b_{2}}, \cdots, s r^{b_{t}}\right\}$,

and $G=\operatorname{Cay}\left(D_{2 n}, \Omega\right)$. If $d_{1}=a_{1}=1, d_{i}=a_{i}-a_{i-1}$ for $2 \leq i \leq k, \quad d_{1}^{\prime}=b_{11}, d_{j}^{\prime}=b_{j}-b_{j-1}$ for $2 \leq j \leq t$ and

$d=\max _{1 \leq i \leq k, 1 \leq j \leq t}\left\{d_{i}, d_{j}^{\prime}\right\}$, then $\gamma_{c}(G) \leq 2 d \frac{n}{a_{k+d-1}}$.

Proof. Let $x=a_{k}+d-1$ and $l=\left\lceil\frac{n}{x}\right\rceil$. Consider the set

$$
S_{t}=\left\{r^{i x+g}, s r^{n-\left(i x+g-b_{1}\right)}: 0 \leq i \leq l-1 \text { and } 0 \leq g \leq d-1\right\} .
$$

In the notation of Lemma 5, $a_{1}=1$ and $x=a_{k}+d-1$ and $S_{c}$ is a total dominating set. Since $r \in \Omega$ and for each $i$ with $0 \leq i \leq l-1$, we have paths $r^{i x}, r^{i x+1}, \cdots, r^{i x+(d-1)}$ and $s r^{n-\left(i x-b_{1}\right)}, s r^{n-\left(i x+1-b_{1}\right)}, \cdots, s r^{n-\left(i x+d-1-b_{1}\right)}$. Also note that $r^{i x+(d-1)}$ and $r^{i x+(d-1)+a_{k}}=r^{(i+1) x}, s r^{n-\left(i x+d-1-b_{1}\right)}$ and $s r^{n-\left(i x+d-1+a_{k}-b_{1}\right)}=s r^{n-\left((i+1) x-b_{1}\right)}$ are connected. Hence the induced subgraph $\left\langle S_{c}\right\rangle$ is connected.

\section{Subgroups as Efficient Domination Sets}

In this section, we obtain some E-sets in $G=\operatorname{Cay}\left(D_{2 n}, \Omega\right)$. Moreover we have identified certain subgroups of $D_{2 n}$ which are also efficient domination sets in $G$.

Theorem 7 Let $n \geq 3$ be an integer, $m=\left\lfloor\frac{n-1}{2}\right\rfloor$ and $k, t$ be integers such that $1 \leq k \leq m, 1 \leq t \leq n$ and $d$ is an integer such that $d(2 k+t+1)$ divides $n$. Let

$$
\begin{aligned}
\Omega= & \left\{r^{d}, r^{2} d, \cdots, r^{k} d, r^{(n-k d)}, r^{n-(k-1) d}, \cdots,\right. \\
& \left.r^{(n-d)}, s r^{d}, s r^{2} d, \cdots, s r^{t} d\right\}
\end{aligned}
$$

and $G=\operatorname{Cay}\left(D_{2 n}, \Omega\right)$. Then $\gamma(G)=\frac{2 n}{2 k+t+1}$. In this case, $G$ has an E-set.

Proof. Let $l=\frac{2 n}{d(2 k+t+1)}$ and $x=d(2 k+t+1)$. In the notation of Lemma $4, d_{i}$ 's and $d_{i}^{\prime}$ 's are same, $a_{i}=i d$ for all $1 \leq i \leq k$ and $b_{j}=j d$ for all $1 \leq j \leq t$. Let $x=d(2 k+t+1)$ and $l=\left\lceil\frac{n}{x}\right\rceil$. By Lemma 4,

$$
S=\left\{r^{i x+g}, s r^{n-(k d+i x+g)}: 0 \leq i \leq l-1,0 \leq g \leq d-1\right\}
$$

is a dominating set and hence $\gamma(G) \leq \frac{2 n}{2 k+t+1}$. Since $G$ is $2 k+t$ regular, by Theorem 1 , one can conclude that $S$ is an E-set in $G$.

Remark 8 Note that Theorem 3 identifies all subgroups of the dihedral group $D_{2 n}$. Now we us identify some of the subgroups as efficient dominating sets.

Theorem 9 Let $n \geq 3$ be an integer, $m=\left\lfloor\frac{n-1}{2}\right\rfloor$ and $k$, $t$ be integers such that $1 \leq k \leq m, 1 \leq t \leq n$ and $2 k+t+1$ divides $n$. Let $H=\left\langle r^{a}, s r^{n-b}\right\rangle$ be a subgroup of the dihedral group $D_{2 n}$, where $a=2 k+t+1$ and $b$, $0 \leq b \leq k-1$ Then, there exists a generating set $\Omega$ of $D_{2 n}$ such that $H$ is an efficient dominating set for the Cayley graph $G=\operatorname{Cay}\left(D_{2 n}, \Omega\right)$.

\section{Proof. Let}

$\Omega=\left\{r, r^{2}, \cdots, r^{k}, r^{n-k}, r^{n-(k-1)}, \cdots, r^{n-1}, s r, s r^{2}, \cdots, s r^{t}\right\}$,

$l=\frac{n}{2 k+t+1}$ and $x=2 k+t+1$. By taking $d=1$ in Theorem 7 ,

$$
S=\left\{r^{0}, r^{x}, \cdots, r^{(l-1) x}, s r^{n-k}, s r^{n-(k+x)}, \cdots, s r^{n-(k+(l-1) x)}\right\}
$$

is an efficient dominating set of $G$.

Remark 10 Under the assumptions of Theorem 9, $S . x$ is an efficient dominating set for the Cayley graph $G=\operatorname{Cay}\left(D_{2 n}, \Omega\right)$ for all $x \in D_{2 n}$.

\section{E-Chains in Cayley Graphs}

Theorem 7 and 9 provide a tool to produce E-sets and visualize some of the subgroups as E-sets in $\operatorname{Cay}\left(D_{2 n}, \Omega\right)$. We use this tool to obtain an inclusive E-chain and inductive subgroups E-chain of Cayley graphs on the dihedral group.

Theorem 11 Let $n \geq 3$ be an integer, $m=\left\lfloor\frac{n-1}{2}\right\rfloor$ and $k$ be an integers such that $1 \leq k \leq m$, $G_{0}=\operatorname{Cay}\left(D_{2 n}, D_{2 n}-\{e\}\right)$, $\Omega_{i}=\left\{r, r^{2}, \cdots, r^{k}, r^{n-k d}, r^{n-(k-1)}, \cdots, r^{n-1}, s r, s r^{2}, \cdots, s r^{n-b_{i}}\right\}$ and $G_{i}=\operatorname{Cay}\left(D_{2 n}, \Omega_{i}\right)(i \geq 1)$ Assume that $\left|\Omega_{i}\right|+1$ divides $n$ and $\left|\Omega_{i+1}\right|+1$ divides $\left|\Omega_{i}\right|+1$. Then the finite family of graphs $\mathbb{G}=\left\{G_{i}, i \geq 0\right\}$ is inductive subgroups E-chain.

Proof. Let $\lambda_{i}=\left|\Omega_{i}\right|+1$. By the assumption $\lambda_{i+1}$. divides $\lambda_{i}$. Define the map $\zeta_{i}: V\left(G_{i}\right) \rightarrow V\left(G_{i+1}\right)$ by $\zeta_{i}(v)=v$ for all $v \in G_{i}$. By Theorem 9, $G_{i}$ has an efficient dominating set and it is of the form

$$
\begin{gathered}
S_{i}=\left\{r^{0}=e, r^{\lambda_{i}}, r^{2 \lambda_{i}}, \cdots, r^{\left(\frac{n}{\lambda_{i}}-1\right) \lambda_{i}}, s r^{n-\left(k+\lambda_{i}\right)},\right. \\
\left.s r^{n-k}, s r^{n-\left(k+2 \lambda_{i}\right)}, \cdots, s r^{n-\left(k+\left(\frac{n}{\lambda_{i}}-1\right) \lambda_{i}\right)}\right\}
\end{gathered}
$$


and also $S_{i}$ 's are subgroups. It implies that $\zeta_{i}\left(S_{i}\right) \subseteq S_{i+1}$ for every $i \geq 1$. Hence the family of graphs $\mathbb{G}=\left\{G_{i}, i \geq 0\right\}$ is inductive subgroups E-chain.

The construction of an inclusive E-chain of Cayley graphs is based on the following lemma.

Lemma 12 Let $n \geq 3$ be an integer, $m=\left\lfloor\frac{n-1}{2}\right\rfloor, k, t$ be integers such that $1 \leq k \leq m, 1 \leq t \leq n$ and $d$ is an integer such that $d(2 k+t+1)$ divides $n$. For $i \geq 1$, let

$$
\begin{aligned}
\Omega_{i}= & \left\{r^{d}, r^{2} d, \cdots, r^{k} d, r^{2^{i} n-d}, r^{2^{i} n-2 d}, \cdots,\right. \\
& \left.r^{2^{i} n-k d}, s r^{d}, s r^{2} d, \cdots, s r^{t} d\right\}
\end{aligned}
$$

and $G_{i}=\operatorname{Cay}\left(D_{2^{i} n}, \Omega_{i}\right)$. Then $G_{i+1}$ is a covering of $G_{i}$.

Proof. Define the surjective map

$f_{i}: V\left(G_{i+1}\right) \rightarrow V\left(G_{i}\right)$ by $f_{i}\left(r^{j}\right)=r^{j \bmod 2^{i} n}$ and

$f_{i}\left(s r^{j}\right)=s r^{j \bmod 2^{i} n}$ for all $j$, where $0 \leq j \leq 2^{i+1}(n-1)$.

Note that $f_{i}$ is a group homomorphism from $D_{2^{i+1} n}$ onto $D_{2^{i} n}$. Let $\tilde{u}, \tilde{v} \in G_{\mathrm{i}+1}$. Suppose $\tilde{u}$ and $\tilde{v}$ are adjacent in $G_{i+1}$. Then, there exists $r^{k}$ with

$1 \leq k \leq\left\lfloor\frac{n-1}{2}\right\rfloor$ or $s r^{t}$ with $1 \leq t \leq n-1$ such that

$\tilde{u}=\tilde{v} . r^{k}$ or $\tilde{u}=\tilde{v} \cdot s r^{k}$. Since $f_{i}$ is a group homomorphism and

$f_{i}\left(r^{k}\right)=r^{k \bmod 2^{i} n}=r^{k}, f_{i}\left(s r^{t}\right)=s r^{t \bmod 2^{i} n}=s r^{t}$, we have $f_{i}(\tilde{u})=f_{i}(\tilde{v}) \cdot r^{k}$ or $f_{i}(\tilde{u})=f_{i}(\tilde{v}) \cdot s r^{t}$ and so $f_{i}(\tilde{u})$ and $f_{i}(\tilde{v})$ are adjacent in $G_{i}$. Consider the map $f_{i} /_{N(\tilde{v})}: N(\tilde{v}) \rightarrow N(v)$ for any vertex $\tilde{v} \in V\left(G_{\mathrm{i}+1}\right)$ and $v \in V\left(G_{i}\right)$. Claim $f_{i} /{ }_{N(\tilde{v})}$ is bijection. Any element $x$ in $N(\tilde{v})$ as either one vertex of the form $x=r^{e}$ or $x=s r^{e}$, where $0 \leq j \leq 2^{i+1}(n-1)$. Let $x, y \in N(\tilde{v})$. Then we have following three cases:

Case 1. Let $x=r^{e_{1}}$ and $y=r^{e_{2}}$ with $e_{1} \neq e_{2}$. Suppose $f_{i}(x)=f_{i}(y)$, i.e.

$r^{e_{1} \bmod 2^{i} n}=r^{e_{2} \bmod 2^{i} n} \Rightarrow r^{\left(e_{1}-e_{2}\right) \bmod 2^{i} n}=e$. i.e.

$o(r)=\left(e_{1}-e_{2}\right) \bmod 2^{i} n<n$, which is a contradiction to $o(r)=n$. Therefore $f_{i}(x) \neq f_{i}(y)$.

Case 2. Let $x=r^{e_{1}}$ and $y=s r^{e_{2}}$. Suppose

$f_{i}(x)=f_{i}(y)$, i.e. $r^{e_{1} \bmod 2^{i} n}=s r^{e_{2} \bmod 2^{i} n}$ This means

$r^{\left(e_{1}-e_{2}\right) \bmod 2^{i} n}=e$ or $s=s r^{\left(e_{1}-e_{2}\right) \bmod 2^{i} n}=e$, which is a contradiction. Therefore $f_{i}(x) \neq f_{i}(y)$.

Case 3. Let $x=s r^{e_{1}}$ and $y=s r^{e_{2}}$ with $e_{1} \neq e_{2}$. Suppose $f_{i}(x)=f_{i}(y)$, i.e.

$s r^{e_{1} \bmod 2^{i} n}=s r^{e_{2} \bmod 2^{i} n} \Rightarrow r^{\left(e_{1}-e_{2}\right) \bmod 2^{i} n}=e$. i.e.

$o(r)=\left(e_{1}-e_{2}\right) \bmod 2^{i} n<n$ which is a contradiction. Therefore $f_{i}(x) \neq f_{i}(y)$. Hence distinct elements of $N(\tilde{v})$ are distinctly mapped onto $N(v)$ and so $f_{i} /_{N(\tilde{v})}$ is a required bijection.
Theorem 13 Let $n \geq 3$ be an integer, $m=\left\lfloor\frac{n-1}{2}\right\rfloor$, $k, t$, be integers such that $1 \leq k \leq m, 1 \leq t \leq n$ and $d$ is an integer such that $d(2 k+t+1)$ divides $n$. For $i \geq 1$ let

$$
\begin{aligned}
\Omega_{i}= & \left\{r^{d}, r^{2} d, \cdots, r^{k} d, r^{2^{i} n-d}, r^{2^{i} n-2 d}, \cdots,\right. \\
& \left.r^{2^{i} n-k d}, s r^{d}, s r^{2} d, \cdots, s r^{t} d\right\}
\end{aligned}
$$

and $G_{i}=\operatorname{Cay}\left(D_{2^{i} n}, \Omega_{i}\right)$. Let $S_{i}$ be an efficient dominating set for $G_{i}$. Then the finite family of graphs $\mathbb{G}=\left\{G_{i}, i \geq 1\right\}$ is an inclusive E-chain.

Proof. Since by above Lemma, $G_{i+1}$ is a covering of $G_{i},(i \geq 1)$. Since by Theorem $2, f_{i}^{-1}\left(S_{i}\right) \subset S_{i+1}$. Hence the finite family of graphs $\mathbb{G}=\left\{G_{i}, i \geq 1\right\}$ is an inclusive E-chain.

\section{Acknowledgements}

The work reported here is supported by the Special Assistance Programme (F510-DRS-I/2007) of University Grants Commission, India awarded to the Department of Mathematics, Manonmaniam Sundaranar University for the period 2007-2012.

\section{REFERENCES}

[1] S. Lakshmivarahan, J. S. Jwo and S. K. Dhall, "Symmetry in Interconnection Networks Based on Cayley Graphs of Permutation Groups: A Survey," Parallel Computing, Vol. 19, No. 4, 1993, pp. 361-407. doi:10.1016/0167-8191(93)90054-O

[2] E. J. Cockayne, R. M. Dawes and S. T. Hedetniemi, "Total Domination in Graphs," Networks, Vol. 10, No. 3, 1980, pp. 211-219. doi:10.1002/net.3230100304

[3] I. J. Dejter and O. Serra, "Efficient Dominating Sets in Cayley Graphs," Discrete Applied Mathematics, Vol. 129, No. 2-3, 2003, pp. 319-328. doi:10.1016/S0166-218X(02)00573-5

[4] R. J. Huang and J.-M. Xu, "The Bondage and Efficient Domination of Vertex Transitive Graphs," Discrete Mathematics, Vol. 308, No. 4, 2008, pp. 571-582. doi:10.1016/j.disc.2007.03.027

[5] J. Lee, "Independent Perfect Domination Sets in Cayley Graphs," Journal of Graph Theory, Vol. 37, No. 4, 2000, pp. 219-231.

[6] T. Tamizh Chelvam and I. Rani, "Dominating Sets in Cayley Graphs on $Z_{n}$," Tamkang Journal of Mathematics, Vol. 37, No. 4, 2007, pp. 341-345.

[7] T. Tamizh Chelvam and I. Rani, "Independent Domination Number of Cayley Graphs on $Z_{n}$," The Journal of Combinatorial Mathematics and Combinatorial Computing, Vol. 69, 2009, pp. 251-255.

[8] T. Tamizh Chelvam and I. Rani, "Total and Connected 
Domination Numbers of Cayley Graphs on $Z_{n}$," $A d$ vanced Studies in Contemporary Mathematics, Vol. 20, 2010, pp. 57-61.

[9] T. W. Haynes, S. T. Hedetniemi and P. J. Slater, "Fundamentals of Domination in Graphs," Marcel Dekker,
New York, 1998.

[10] K. Conrad, "Dihedral Groups II,” 2009. http://www.math.uconn.edu/ kconrad/blurbs/grouptheory /dihedral2.pdf 